\title{
Síndrome de Stevens-Johnson y necrólisis epidérmica tóxica en el Hospital Universitario del Valle "Evaristo García" durante un periodo de 9 años
}

Stevens Johnson syndrome and toxic epidermal necrolysis at the Hospital Universitario del Valle "Evaristo García” for a period of 9 years.

\section{David Andrés Arias' ${ }^{1}$, Paola Andrea Londoño², Juana Gabriela López², Luis Hernando Moreno ${ }^{3}$}

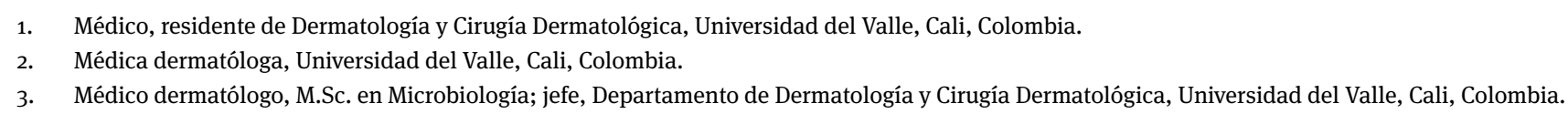

\section{Resumen}

La necrólisis epidérmica tóxica y el síndrome de Stevens-Johnson son las reacciones cutáneas a medicamentos de mayor gravedad y constituyen un espectro de la misma enfermedad, compartiendo aspectos etiológicos, patogénicos, histológicos y terapéuticos.

Objetivos. Describir los casos de necrólisis epidérmica tóxica y síndrome de Stevens-Johnson durante un periodo de nueve años en el Hospital Universitario del Valle, los tratamientos usados en el manejo de los pacientes y la mortalidad de los mismos.

Metodología. Se hizo una revisión retrospectiva de las historias clínicas de los pacientes con Stevens-Johnson y necrólisis epidérmica tóxica, diagnosticados y manejados desde febrero de 2003 hasta abril de 2012 en el Hospital Universitario del Valle. Se analizaron las características clínicas, frecuencia, mortalidad, medicamentos relacionados como posibles desencadenantes, tratamientos utilizados y sus resultados.

Resultados. Se analizaron los datos de 29 casos, 13 (44,8 \%) tenían diagnóstico de necrólisis epidérmica tóxica, 13 (44,8 \%) de síndrome de Stevens-Johnson y los tres restantes (10,3 \%) síndrome de superposición de síndrome de StevensJohnson y necrólisis epidérmica tóxica. En el $55 \%(n=16)$ se pudo establecer un solo medicamento como desencadenante. En $41 \%(n=12)$ de los pacientes se encontraron dos o más medicamentos como posibles desencadenantes. Solo en uno no se identificó ningún medicamento. Los antibióticos, los anticonvulsivos, los AINE y el alopurinol fueron los más asociados. Todos los pacientes recibieron medidas de soporte. El $68 \%(\mathrm{n}=20)$ de los pacientes recibió tratamiento antibiótico debido a infección asociada. Veintitrés pacientes (79,3\%) recibieron pentoxifilina como parte de su tratamiento. Seis $(20,6 \%)$ recibieron gammaglobulina (dos de ellos asociada a pentoxifilina oral). La mortalidad fue del 3,4 \% (n=1).

Conclusiones. Las medidas de soporte son fundamentales en el manejo de los pacientes con necrólisis epidérmica tóxica y síndrome de Stevens-Johnson, independientemente del área corporal comprometida, así como el manejo oportuno de complicaciones como la infección sobreagregada. La pentoxifilina puede considerarse una alternativa en el tratamiento de estas enfermedades.

\section{Correspondencia:}

David Andrés Arias

Email:

davidandresarias@hotmail.com

Recibido: 20 de junio de 2013.

Aceptado: 21 de agosto de 2013.

No se reportan conflictos de intereses. 
PALABRAS CLAVE: síndrome de Stevens-Johnson, necrólisis epidérmica tóxica, factor de necrosis tumoral alfa, pentoxifilina.

\section{Summary}

The Stevens-Johnson syndrome and the toxic epidermal necrolysis are very severe cutaneous drug reactions and constitute a spectrum of the same disease, sharing etiological, pathogenic, histological and therapeutic aspects.

Objectives: To describe the cases of toxic epidermal necrolysis and Stevens-Johnson syndrome over a nine year period at the Hospital Universitario del Valle, the treatments used in these patients and their mortality.

Methodology: A medical history review was carried out retrospectively of patients with Stevens-Johnson syndrome and toxic epidermal necrolysis diagnosed and managed from February, 2003, until April, 2012, at the Hospital Universitario del Valle. We analyzed clinical characteristics, frequency, mortality, medications as possible triggers, treatments used and response to them.

Results: Out of 29 cases, 13 (44.8\%) had diagnosis of toxic epidermal necrolysis, $13(44.8 \%)$ had Stevens-Johnson syndrome and the remaining 3 (10.3\%), overlapping Stevens-Johnson syndrome-toxic epidermal necrolysis. In 16 patients $(55 \%)$ a single drug could be the trigger. In 12 patients (41\%) two or more drugs were found as possible triggers. Drugs were identified only in one. Antibiotics, anticonvulsants, NSAIDs and allopurinol were the most frequently associated drugs. All patients received supporting measures. Twenty patients (68\%) received antibiotic therapy due to an associated infection. Twenty three patients $(79.3 \%)$ received pentoxifylline as part of their treatment; six (20.6\%) received gammaglobulin (two of them associated with oral pentoxifylline). One patient died (3.4\%).

Conclusions: The supporting measures are essential in the management of patients with toxic epidermal necrolysis and Stevens- Johnson syndrome independent of the affected body area, as well as the timely management of complications as superinfection. Pentoxifylline may be considered an alternative in the treatment of these pathologies.

KEY WORDS: Stevens Johnson syndrome, toxic epidermal necrolysis, tumor necrosis factor alpha, pentoxifylline.

\section{Introducción}

Las reacciones cutáneas a medicamentos ocurren hasta en el $2 \%$ de todos los tratamientos instaurados a nivel mundial ${ }^{1}$ La necrólisis epidérmica tóxica y el síndrome de Stevens-Johnson, son las reacciones cutáneas a medicamentos de mayor gravedad y constituyen un espectro de la misma enfermedad, compartiendo aspectos etiológicos, patogénicos, histológicos y terapéuticos² ${ }^{2}$.

La necrólisis epidérmica tóxica y el síndrome de Stevens-Johnson son reacciones idiosincráticas y exfoliativas de la piel y las mucosas ${ }^{3}$, de presentación infrecuente (incidencias entre 1 y 2 casos por millón de personas para la necrólisis epidérmica tóxica y de hasta 7 casos por millón para el síndrome de StevensJohnson $)^{4}$, con una mortalidad de hasta $5 \%$ para el síndrome de Stevens-Johnson ${ }^{5}$ y entre el $25 \mathrm{y}$ el $70 \%$ para la necrólisis epidérmica tóxica, dependiendo de las series $^{1,2,3,4}$. Ambas son ocasionadas principalmente por una reacción al consumo de diferentes medicamentos; también se han descrito aditivos y otros químicos e infecciones, especialmente por herpes simple y Mycoplasma pneumoniae $e^{4,6,7}$. Afectan poblaciones de cualquier raza y edad, aunque comparadas con la de los adultos, las manifestaciones en la niñez suelen ser más leves y con menor mortalidad 5 .

Los medicamentos que se han implicado con mayor frecuencia son: las sulfonamidas (en especial en combi- 
nación con trimetoprim), las penicilinas, los anticonvulsivos y los antinflamatorios no esteroideos ${ }^{4,6,7}$.

El síndrome de Stevens-Johnson difiere de la necrólisis epidérmica tóxica en el porcentaje de superficie corporal afectada, lo cual tiene importancia en el pronóstico y subdivide este espectro en tres grupos. En el síndrome de Stevens-Johnson, se afecta menos del $10 \%$ de la superficie corporal y, en la necrólisis epidérmica tóxica, más del $30 \%$; los casos intermedios, con un compromiso entre el 10 y el $30 \%$, se clasifican como una superposición síndrome de Stevens-Johnson-necrólisis epidérmica tóxica.

Clínicamente se inician con fiebre y malestar general simulando una infección respiratoria alta $^{8}$ y posteriormente, presentan intenso eritema en algunas zonas de la piel, acompañada de pápulas o placas que luego se ampollan y se esfacelan fácilmente (signo de Nikolsky) y progresan hasta comprometer grandes áreas de la superficie corporal. Las lesiones están distribuidas simétricamente, iniciándose comúnmente en la cara o en la parte alta del tronco; las mucosas oral, nasal, genital y anal, están comprometidas en un gran porcentaje de pacientes y en algunos se ha descrito afectación respiratoria y gastrointestinal ${ }^{9,10}$.

La patogénesis no se encuentra completamente entendida. El principal fenómeno es básicamente una apoptosis masiva de las células de la capa epidérmica. Se ha planteado que sea producida por las caspasas, que son una familia de proteasas activadas por la unión de ligandos a receptores de muerte, o que sea causada por daño mitocondrial resultante de la perturbación del balance entre proteínas proapoptóticas y antiapoptóticas, y por las granzimas, que son serinproteasas presentes en los gránulos de los linfocitos T citotóxicos y asesinos naturales (natural killer), que pueden inducir apoptosis después de la unión de las perforinas ${ }^{11}$, ya sea por vía de las caspasas o de manera independiente ${ }^{10}$.

En síntesis, lo que se plantea es que en individuos vulnerables ${ }^{4}$, el consumo del medicamento estimula una expansión de linfocitos T CD8 que inician los mecanismos proapoptóticos de manera directa o indirecta ${ }^{10}$, además del aumento de citocinas proinflamatorias ${ }^{12}$.

De acuerdo con lo anterior, los tratamientos encontrados en la literatura científica para ambos cuadros incluyen: identificar y descontinuar el fármaco causante (se plantea incluso la plasmaféresis), proporcionar medidas eficientes de soporte ${ }^{13}$ (mantener el equilibrio hidroelectrolítico y metabólico, y proteger la dermis expuesta y las mucosas erosionadas), establecer medidas que eviten la sobreinfección y la administración de medicamentos específicos como inmunosupresores (ciclosporina), agentes antiapoptóticos (inmunoglobulina humana administrada de forma endovenosa ${ }^{14}$ ), y los anti-TNF (medicamentos biológicos ${ }^{10}$ ), sin que haya unificación de criterios sobre el tratamiento ${ }^{10,15}$.

En el Hospital Universitario del Valle se presentan casos de síndrome de Stevens-Johnson y de necrólisis epidérmica tóxica, sin que en la actualidad se conozca su frecuencia, cuáles son los medicamentos que más se asocian con ellos, cuál es la mortalidad y, al igual que en la literatura mundial, aún no hay un consenso de qué tratamiento específico se debe usar $^{10}$.

\section{Materiales y métodos}

Se hizo una revisión retrospectiva de las historias clínicas de los pacientes con síndrome de Stevens-Johnson y necrólisis epidérmica tóxica, diagnosticados y manejados desde febrero de 2003 hasta abril de 2012, en el Hospital Universitario del Valle. Se incluyeron pacientes de cualquier edad y sexo y se excluyeron aquellos en los que el diagnóstico no era claro, no contaban con valoración por dermatología o tenían historia clínica incompleta que no permitía obtener los datos completos respecto a la enfermedad en estudio.

Se registró en un formato realizado por los investigadores, la edad, el sexo, la raza, los medicamentos desencadenantes, el diagnóstico, los tratamientos recibidos y su duración, así como la evolución de cada uno de los pacientes hasta su egreso o muerte. Los datos se analizaron en el paquete estadístico STATA ${ }^{\circledR}$ 10.0. Para el análisis se utilizaron medidas de tendencia central y dispersión. La medida resumen para las variables discretas fue la proporción. Para las variables continuas, se usaron el promedio y la desviación estándar.

\section{Resultados}

Se analizaron los datos de 29 casos: 14 mujeres (48,2 \%) y 15 hombres $(51,8 \%)$. La mediana de edad fue de 27 años (rango, 1 a 77 ) y 11 (38\%) tenían menos de 18 años. Trece $(44,8 \%)$ tenían diagnóstico de necrólisis epidérmica tóxica, $13(44,8 \%)$ síndrome de Stevens-Johnson y, los tres restantes (10,3\%) síndrome de superposición de síndrome de Stevens-Johnson y necrólisis epidérmica tóxica (FIGURA 1).

En 16 casos (55\%) se pudo establecer un solo medicamento como desencadenante, de los cuales 6 correspondieron a fenitoína, 5 a antibióticos ( 2 a trimetoprimsulfametoxazol, 1 a vancomicina, 1 a azitromicina, 1 a amoxicilina), 3 a AINE (1 no especificado, 1 a dipirona, 1 a diclofenaco) 1 a alopurinol y 1 a sulfato ferroso. En $12(41 \%)$ de los pacientes se encontraron dos o más medicamentos como posibles desencadenantes. En este grupo, los antibióticos, anticonvulsivos, AINE y 


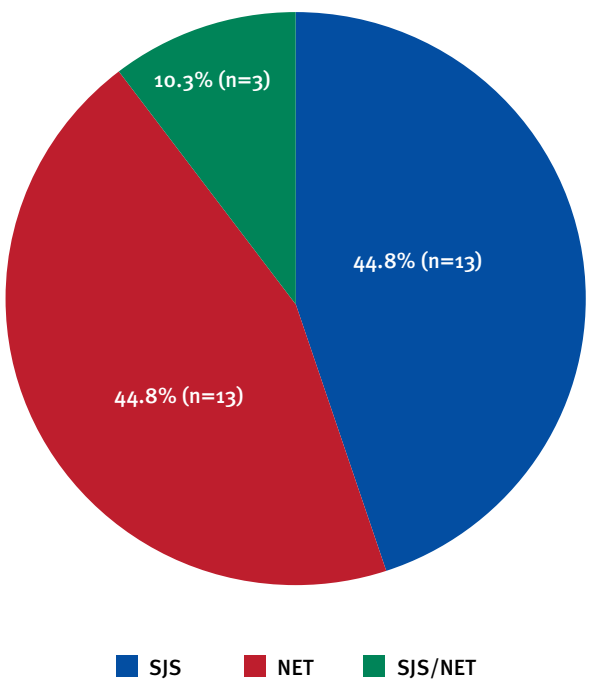

FiguRA 1. Distribución de casos según diagnóstico, Hospital Universitario del Valle, febrero de 2003 a abril de 2012 (n=29)

alopurinol fueron los que más se encontraron. Solo en uno no se identificó ningún medicamento. En cuanto al manejo (figura 2), todos recibieron medidas de soporte (administración de líquidos endovenosos, soporte nutricional, cuidados de piel que incluían curaciones con solución salina $0,9 \%$ y cubrimiento con gasas vaselinadas de las zonas afectadas) y $8(27,5 \%)$ requirieron terapia respiratoria.

En los 28 casos (96,5\%) en que se identificó un fármaco como posible desencadenante, este se suspendió de inmediato. Veinte pacientes $(69 \%)$ recibieron tratamiento antibiótico debido a la presencia de infección asociada. Veintitrés pacientes $(79,3 \%)$ recibieron pentoxifilina como parte de su tratamiento (rango, 1 a 60 días), de los cuales, 20 recibieron pentoxifilina endovenosa $(0,6 \mathrm{mg} /$ $\mathrm{kg}$ por hora en infusión con un rango de 1 a 30 días), incluido el paciente fallecido, y a 7 de ellos, al presentar mejoría de las lesiones en piel, se les cambió a pentoxifilina oral (400 mg cada 8 horas con un rango de 4 a 45 días); solo 3 pacientes de este grupo recibieron pentoxifilina oral sin previo uso de pentoxifilina endovenosa (2 de ellos habían recibido gammaglobulina). De todos los casos analizados, $6(20,6 \%)$ recibieron gammaglobulina (rango, 2 a 5 días), 2 de ellos recibieron gammaglobulina por 5 días y luego se continuó con pentoxifilina oral por 6 a 10 días más.

Ocho $(27,5 \%)$ casos recibieron, además de lo descrito, esteroides sistémicos administrados en forma intermitente: tres de ellos el primer día de ingreso hospitalario, otros tres entre 3 y 7 días desde el ingreso y dos lo recibieron por más de una semana. Uno de ellos lo venía re-

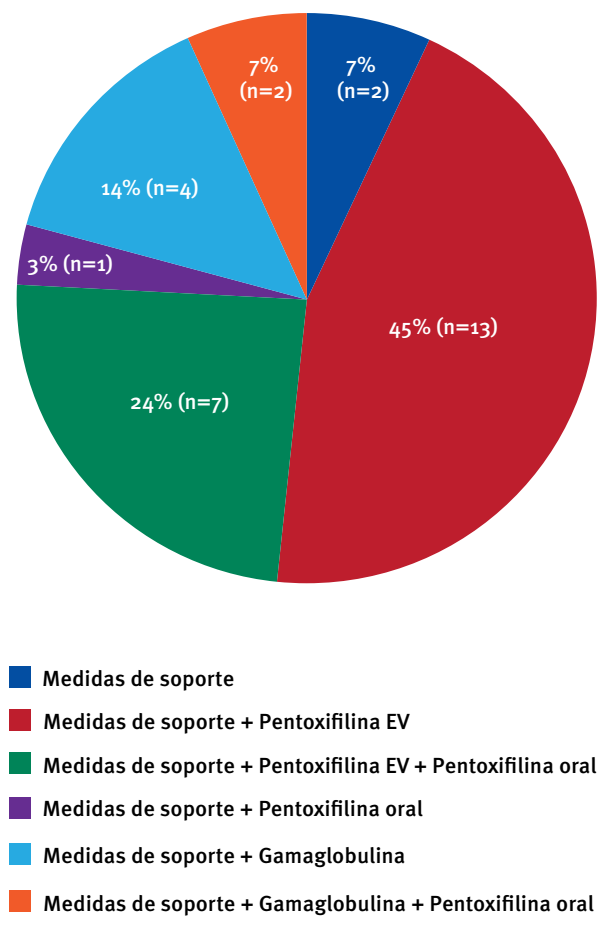

FIGURA 2. Distribución de los tratamientos de pacientes con síndrome de Stevens-Johnson y necrólisis epidérmica tóxica, Hospital Universitario del Valle, febrero de 2003 a abril de $2012(n=29)$.

cibiendo un mes antes del ingreso hospitalario, porque se creía que tenía un pénfigo foliáceo, y el restante lo recibió antes del inicio del cuadro y durante toda su hospitalización, por una enfermedad del colágeno de base. La mortalidad fue del 3,4 \% (n=1).

\section{Discusión}

El síndrome de Stevens-Johnson y la necrólisis epidérmica tóxica son dos espectros de una misma entidad que se caracterizan por el compromiso cutáneo (desprendimiento) y de mucosas, precedidos de malestar general y fiebre ${ }^{8,16}$ cuya patogenia no se encuentra totalmente entendida. Se ha descrito una serie de moléculas que podrían tener participación en el desarrollo de las manifestaciones clínicas, como la granulisina ${ }^{17}$, las perforinas, la granzima B y los mediadores inflamatorios, entre ellos el TNF-alfa ${ }^{12,18}$.

La incidencia reportada en la literatura científica es de 2 a 7 casos por millón por año $0^{4,19}$. La mortalidad en este estudio fue de 3,4\% (n=1); este paciente presentó necrólisis epidérmica tóxica y tenía VIH en estadio de Sida, y desarrolló una infección por Enterococcus spp. aso- 
ciado al cuadro clínico. Comparada con la informada en la literatura científica ${ }^{3,5,20,21,}$ esta mortalidad es inferior; esto podría explicarse por el manejo oportuno de las infecciones que los pacientes presentaron así como los cuidados de piel, el control estricto de líquidos y electrolitos y el soporte nutricional.

El principal factor desencadenante fue el consumo de medicamentos, en $96,5 \%(n=28)$, lo cual es similar con lo descrito en la literatura científica ${ }^{4,6,7}$. Se encontró que los antibióticos, los anticonvulsivos (especialmente la fenitoína), los AINE y el alopurinol, fueron los principales medicamentos desencadenantes.

En cuanto al tratamiento, todos recibieron medidas de soporte independientemente del área corporal comprometida y no se determinó el Severity-of-illness Score for Toxic Epidermal Necrolysis (SCORTEN) ${ }^{22,23}$. Según lo reportado en la literatura científica, el retiro del medicamento sospechoso como causante del cuadro clínico ${ }^{24}$ y las medidas de soporte son el pilar del tratamiento ${ }^{25}$. Las medidas de soporte consistieron en la reposición de líquidos y electrolíticos, soporte nutricional, terapia respiratoria, cuidados de la piel cubriendo el área comprometida con gasas recubiertas con vaselina y curaciones con solución salina. Todos los pacientes fueron manejados conjuntamente con otras especialidades.

El 79,3 \% recibió esquema de tratamiento con pentoxifilina. La información de su uso en este tipo de pacientes es escasa y, al parecer, se han encontrado beneficios en reportes de $\operatorname{casos}^{26,27}$. En nuestra institución se ha tenido experiencia con el uso de la pentoxifilina en estos casos, lo cual fue publicado en un estudio donde 9 niños con síndrome de Stevens-Johnson y necrólisis epidérmica tóxica recibieron pentoxifilina intravenosa asociada a medidas de soporte y antibióticos, y se presentó una sola muerte ${ }^{28}$. La pentoxifilina es una metilxantina que posee funciones inmunomoduladoras, tales como la inhibición de la producción de TNF alfa por parte de los monocitos y la disminución en la reacción de los leucocitos a dicha citocina y a la interleucina $1^{28,29}$, razón por la cual se podría explicar su utilidad en el manejo de estos pacientes ${ }^{28}$. También se han hecho estudios con inmunoglobulina intravenosa que han sugerido beneficios en el tratamiento de estas enfermedades ${ }^{14,30,31}$. En nuestro estudio se usó este medicamento en seis pacientes.

El 27,5\% $(n=8)$ de los pacientes recibió manejo con esteroides en pulsos cortos; seis lo recibieron por menos de una semana y los dos restantes por más tiempo; ninguno de ellos se asoció con mortalidad, en contraste con lo reportado en la literatura científica por algunos autores $^{32,33}$. No se pudo determinar el SCORTEN ya que ningún paciente tenía registradas todas las variables clínicas y de laboratorio necesarias para tal $\mathrm{fin}^{22,23}$.

Debido a la mortalidad de 3.4\%y la utilización de pen- toxifilina en el $79.3 \%(n=23)$ en el presente estudio, con una sola muerte entre los pacientes que la recibieron pero no atribuible al mismo debido a las condiciones adicionales que el paciente presentaba, asociado a la experiencia reportada previamente en nuestra institución con el uso de dicho medicamento ${ }^{28}$ y teniendo en cuenta la mortalidad registrada por otros investigadores en pacientes que solo recibieron medidas de soporte $(25 \%)^{25}$,es importante llevar a cabo más estudios sobre el uso de la pentoxifilina en estos pacientes para determinar si realmente tiene impacto sobre la disminución en la mortalidad.

\section{Conclusiones}

Las medidas de soporte son fundamentales y necesarias en el manejo de los pacientes con necrólisis epidérmica tóxica y síndrome de Stevens-Johnson, independientemente del área corporal comprometida, así como el tratamiento oportuno de complicaciones, especialmente las infecciones y el desequilibrio hidroelectrolítico. La pentoxifilina se puede considerar como una alternativa en el tratamiento de este tipo de enfermedades.

\section{Agradecimientos}

Al Dr. Christian Pallares de Epidemiología Hospitalaria del Hospital Universitario del Valle.

\section{Referencias}

1. Cross A, Borges S, Estévez F. Reacciones adversas medicamentosas graves: síndrome de Stevens-Johnson y necrólisis epidérmica tóxica. Rev Med Urug. 2004;20:172-7.

2. García I, Roujeau JC, Cruces M. Necrólisis epidérmica tóxica y síndrome de Stevens-Johnson: clasificación y actualidad terapéutica. Actas Dermosifiliogr. 2000;91:541-51.

3. Criton S, Devi K, Sridevi RK, Asokan RU. Toxic epidermal necrolysis: A retrospective study. Int J Dermatol. 1997;36:923-5.

4. Knowles S, Sheir N. Clinical risk management of Stevens-Johnson syndrome/toxic epidermal necrolysis spectrum. Dermatol Ther. 2009;22:441-51.

5. Jean-AanKoh M, KwangTay Y. An update on Stevens-Johnson syndrome and toxic epidermal necrolysis in children. Curr Opin Pediatr. 2009;21:505-10.

6. 6. Akosa AB, Elhag AM. Toxic epidermal necrolysis. A study of the sweat glands. J Clin Pathol. 1995;22:359-64.

7. Ioannides D, Vakali G, Chrysomallis F, Chaidemenos G, Mpatsios $\mathrm{K}$, Mourellou O, et al. Toxic epidermal necrolysis: A study of 22 cases. J Eur Acad Dermatol Venereol. 1994;3:266-75.

8. Weightman W. Toxic epidermal necrolysis. Australas J Dermatol. 1996;37;167-77.

9. Gerdts B, Vloemans AFPM, Kreis RW. Toxic epidermal necrolysis; 15 years experience in a Dutch burns centre. J Eur Acad Dermatol Venereol. 2007;21:781-8. 
10. Chave TA, Mortimer NJ, Sladden MJ, Hall AP, Hutchinson PE. Toxic epidermal necrolysis: Current evidence, practical management and future directions. Br J Dermatol. 2005;153:241-53.

11. Lazarczyk M, Grzela T, Korczak-Kowalska G, Niderla J, Oldak M, Jozwiak J, et al. Pentoxifylline inhibits perforin-dependent natural cytotoxicity in vitro. Oncol Rep. 2002;9:423-6.

12. Correia O, Delgado L, Leal I, Campillo F, Fleming-Torrinha J. Increased interleukin 10, tumor necrosis factor $\square$ and interleukin 6 levels in blister fluid of toxic epidermal necrolysis. J Am Acad Dermatol 2002;47:58-62.

13. Parsons J. Toxic epidermal necrolysis. Int J Dermatol. 1992;31;749-68.

14. Tristani-Firouzi P, Petersen M, Saffle J, Morris S, Zone J. Treatment of toxic epidermal necrolysis with intravenous immunoglobulin in children. J Am Acad Dermatol. 2002;47:548-52.

15. Teo L, Tay YK, Liu TT, Kwok C. Stevens-Johnson syndrome and toxic epidermal necrolysis: Efficacy of intravenous immunoglobulin and a review of treatment options. Singapore Med J. 2009;50:29-33.

16. Bastuji-Garin S, Rzany B, Stern RS, Shear NH, Naldi L, Roujeau JC. Clinical classification of cases of toxic epidermal necrolysis, Stevens-Johnson syndrome, and erythema multiforme. Arch Dermatol 1993; 129: 92-6.

17. Chung WH, Hung SI, Yang JY, Su SC, Huang SP, Wei CY et al. Granulysin is a key mediator for disseminated keratinocyte death in Stevens-Johnson syndrome and toxic epidermal necrolysis. Nat Med.2008; 14:1343-50.

18. Posadas SJ, Padial A, Torres MJ, Mayorga C, Leyva L, Sanchez E, et al. Delayed reactions to drugs show levels of perforin, granzyme $\mathrm{B}$, and Fas-L to be related to disease severity. J Allergy Clin Immunol. 2002;109:155-61.

19. 19. Schöpf E, Stühmer A, Rzany B, Victor N, Zentgraf R, Kapp JF. Toxic Epidermal Necrolysis and Stevens-Johnson Syndrome An Epidemiologic Study From West Germany. Arch Dermatol. 1991;127:839-42.

20. Letko E, Papaliodis DN, Papaliodis GN, Daoud YJ, Ahmed AR, Foster CS. Stevens-Johnson syndrome and toxic epidermal necrolysis: a review of the literature. Ann Allergy Asthma Immunol. 2005; 94:419-36.

21. Revuz J, Penso D, Roujeau JC, Guillaume JC, Payne CR, Wechsler J, et al. Toxic epidermal necrolysis. Clinical findings and prognosis factors in 87 patients. Arch Dermatol. 1987;123:1160-5.
22. Bastuji Garin S, Fouchard N, Bertoschi M, Roujeau JC, Revuz J, Wolkenstien P. SCORTEN: A severity-of-illness score for toxic epidermal necrolysis. J Invest Dermatol. 2000;115:149-53.

23. Guégan S, Bastuji-Garin S, Poszepczynska-Guigné E, Roujeau JC, Revuz J. Performance of the SCORTEN during the first five days of hospitalization to predict the prognosis of epidermal necrolysis. J Invest Dermatol 2006;126:272-6

24. Garcia-Doval I, LeCleach L, Bocquet H, Otero XL, Roujeau JC. Toxic epidermal necrolysis and Stevens-Johnson syndrome: does early withdrawal of causative drugs decrease the risk of death? Arch Dermatol. 2000;136:323-7.

25. Schneck J, Fagot JP, Sekula P, Sassolas B, Roujeau JC, Mockenhaupt M. Effects of treatments on the mortality of Stevens-Johnson syndrome and toxic epidermal necrolysis: A retrospective study on patients included in the prospective. EuroSCAR Study. J Am Acad Dermatol. 2008;58:33-40.

26. Redondo P., De Erenchun F.R., Iglesias M.E., Monedero P. Quintanilla E. Toxic epidermal necrolysis. Treatment with pentoxifylline. Br. J. Dermatol.1994; 130: 688-9.

27. Sanclemente G., De la Roche C.A., Escobar C.E., Falabella R. Pentoxifylline in toxic epidermal necrolysis and Stevens-Johnson syndrome. Int. J. Dermatol., 38: 878-9, 1999.

28. Victoria J, Hormaza X. Pentoxifilina en enfermedades catastróficas en niños. Dermatol Pediatr Lat. 2004;2:21-6.

29. Samlaska CP, Winfield EA. Pentoxifylline. J Am Acad Dermatol. 1994;30:603-21.

30. French LE, Trent JT, Kerdel FA. Use of intravenous immunoglobulin in toxic epidermal necrolysis and Stevens-Johnson syndrome: Our current understanding. Int Immunopharmacol. 2006;6:543-9.

31. Prins C, Kerdel FA, Padilla RS, Hunziker T, Chimenti S, Viard I et al. Treatment of toxic epidermal necrolysis with high-dose intravenous immunoglobulins: multi-center retrospective analysis of 48 consecutive cases. Arch Dermatol 2003; 139: 26-32.

32. Halebian P. Improved burn center survival of patients with toxic epidermal necrolysis managed without corticosteroids. Ann Surg. 1986;204:503-12.

33. Roujeau JC, Stern RS. Severe adverse cutaneous reactions to drugs. N Engl J Med. 1994;331:1272-85.

34. Harr T, French LE. Toxic epidermal necrolysis and StevensJohnson syndrome. Orphanet J Rare Dis. 2010;16;39. 